\title{
An Iterative Run-to-Run Learning Model to Derive Continuous Brachial Pressure Estimates from Arterial and Venous Lines During Dialysis Treatment
}

\author{
Jill Stewart ${ }^{\mathrm{a}}$, Paul Stewart ${ }^{\mathrm{a}, \mathrm{e}}$, Tom Walker ${ }^{\mathrm{a}}$, Daniela Viramontes Horner ${ }^{\mathrm{b}, \mathrm{c}}$, \\ Bethany Lucas ${ }^{\mathrm{b}, \mathrm{c}}$, Kelly White ${ }^{\mathrm{c}}$, Maarten W Taal ${ }^{\mathrm{b}, \mathrm{c}}$, Nicholas M Selby ${ }^{\mathrm{b}, \mathrm{c}}$, \\ Mel Morris ${ }^{\mathrm{d}}$ \\ ${ }^{a}$ School of Health and Social Care, University of Derby, UK \\ ${ }^{b}$ Centre for Kidney Research and Innovation, University of Nottingham, Derby, UK \\ ${ }^{c}$ Renal unit, Royal Derby Hospital, Derby, UK \\ ${ }^{d}$ ITREND Medical Research Ltd. UK \\ ${ }^{e}$ Corresponding Author: p.stewart1@derby.ac.uk
}

\begin{abstract}
Objective: Non-invasive continuous blood pressure monitoring is not yet part of routine practice in renal dialysis units but could be a valuable tool in the detection and prevention of significant variations in patient blood pressure during treatment. Feasibility studies have delivered an initial validation of a method which utilises pressure sensors in the extra-corporeal dialysis circuit, without any direct contact with the person receiving treatment. Our main objective is to further develop this novel methodology from its current early development status to a continuous-time brachial artery pressure estimator. Method: During an in vivo patient feasibility study with concurrent measurement validation by Finapres Nova experimental physiological measurement device, real-time continuous dialysis line pressures, and intermittent occluding arm cuff pressure data were collected over the entire period of (typically 4-hour) dialysis treatments. There was found to be an underlying quasilinear relationship between arterial line and brachial pressure measurements which supported the development of a mathematical function to describe the relationship between arterial dialysis line pressure and brachial artery BP. However, unmodeled non-linearities, dynamics and time-varying parameters present challenges to the development of an accurate BP estimation system. In this paper, we start to address the problem of physiological parameter time variance by novel application of an iterative learning run-to-run
\end{abstract}


modeling methodology originally developed for process control engineering applications to a parameterised BP model.

Results: The iterative run-to-run learning methodology was applied to the real-time data measured during an observational study in 9 patients, supporting subsequent development of an adaptive real-time BP estimator. Tracking of patient BP is analysed for all the subjects in our patient study, supported only by intermittent updates from BP cuff measurements.

Conclusion: The methodology and associated technology is shown to be capable of tracking patient BP non-invasively via arterial line pressure measurement during complete 4-hour treatment sessions. A robust and tractable method is demonstrated, and future refinements to the approach are defined.

Keywords: Dialysis, Blood Pressure, Monitoring, Iterative Learning, Run-to-Run Control

\section{Introduction}

Continuous monitoring of blood pressure (BP) during dialysis (1) has the potential to improve patient outcomes $(2 ; 3 ; 4)$ and could ultimately inform a treatment regime or close a personalised therapeutic intervention loop via modulation of dialysis time and/or duration, dialysate sodium concentration and/or temperature on a per patient basis. Of particular interest is intradialytic hypotension (IDH), a decrease in systolic blood pressure (5), generally a relatively sudden event with associated symptoms include dizziness or fainting, muscle cramps, abdominal discomfort, nausea and vomiting, often results in truncated dialysis treatments with increased risk of cerebral ischemic incidents. IDH prediction, detection and mitigation is the primary application area of the work described in this paper.

$\mathrm{BP}$ monitoring is normally conducted in a clinical setting via the use of an air-filled occluding arm cuff that provides a robust, but irregular and intermittent measurement that disrupts the normal blood flow, and consequently requires a significant settling time between measurements (6). Additionally, there are three alternative distinct methods for non-invasive monitoring of $\mathrm{BP}$ which have occasionally been used in research settings. Arterial applanation tonometry (7), where a transducer is positioned above a superficial artery compressing it against an underlying bone (8), has been used in cardiology and anaesthetised procedures to avoid the insertion of an arterial cannula $(9 ; 10)$. Pulse Transition Time (PTT) $(11)$ is based on measured 
photoplestimography (PPG) and electrocardiogram (ECG) signals during several cardiac cycles, which may not be accurate due in part to the unaccounted physiological factors in the blood pressure regulation mechanism and reliance on accurate ECG triggering (12). Finally, the volume clamp (or vascular unloading) method (6) utilises an inflatable finger-cuff combined with an embedded photodiode to measure the diameter of finger artery. Cuff pressure is adjusted to maintain a constant artery diameter, and the changes in cuff pressure are used to calculate a BP curve in the brachial artery. In use, patients frequently report pain or discomfort at the fingertips where cuffs are placed, and this device can be unreliable in patients with reduced blood flow to the digits, often manifest in dialysis patients (11). All three non-invasive methods are sensitive to patient movement (especially ECG signals) (13) which would result in an unacceptable and uncomfortable restriction placed on patients during a four-hour dialysis treatment.

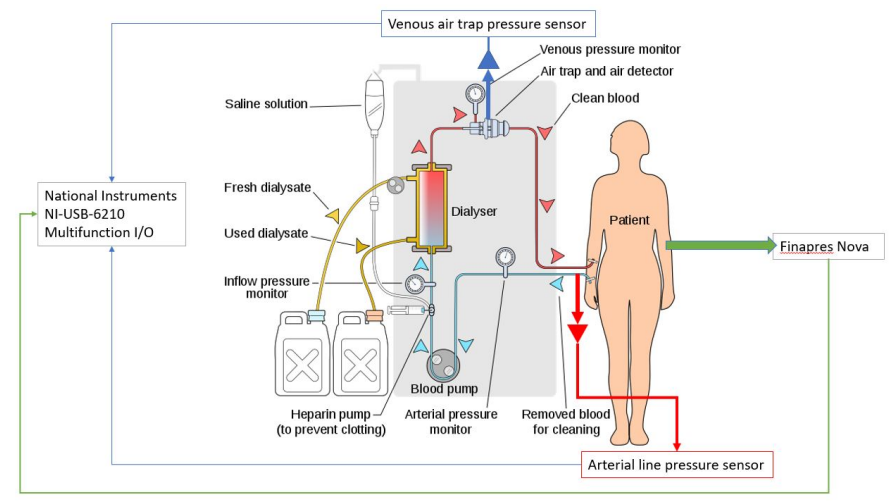

Figure 1: Sensor and data acquisition signal flow

The iTrend (Intelligent Technologies for Renal Dialysis) programme is a long-term collaborative project performed by a multidisciplinary research team from the Universities of Derby and Nottingham and the Royal Derby Hospital Renal Unit in the UK $(24 ; 25)$, developing supporting technologies, real-time analysis algorithms and heuristics to enable personalised and precision treatment in end-stage kidney disease (ESKD) $(14 ; 15)$. Blood pressure monitoring to diagnose hypertension and hypotension $(16 ; 17)$ typically relies on plethorperidialytic and intermittent intradialytic BP measurements. The ability to continuously monitor BP both in terms of absolute measures and blood pressure variability could lead to new diagnostic and prognostic criteria for IDH as well as optimal and personalised treatment strategies. 
A feasibility study of a novel method for continuously monitoring BP has previously been conducted (Fig. 1), which introduced the placement of pressure transducers onto the venous and arterial blood lines that connect a patient to a haemodialysis machine during treatment (18), and experimentally derived relationships between pressures in these extra-corporeal blood lines and brachial BP. Pressure sensors were attached to the blood lines rather than making use of the pressure sensors present within the dialysis machines, and utilised the existing ports and connection points which are common and standardised on most dialysis blood line sets.

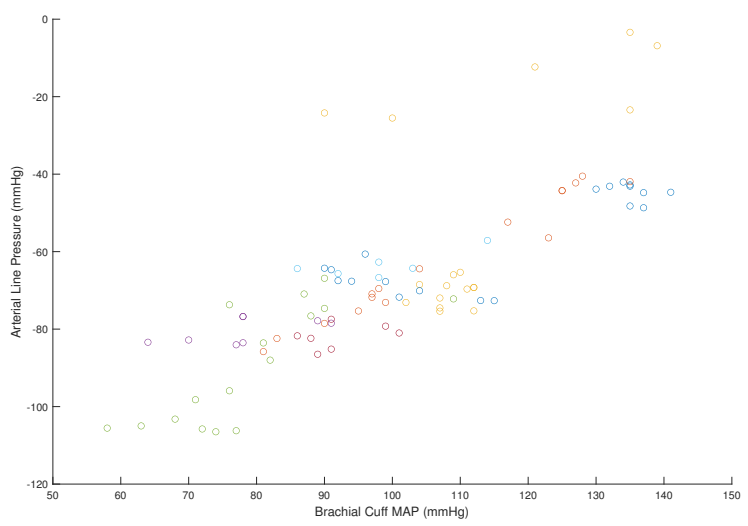

Figure 2: Brachial cuff MAP vs Arterial line pressures in 11 patient sessions

The experimental relationship between brachial cuff MAP and corresponding measured arterial line pressure for 11 sessions in the patient study is shown in Fig. 2, with a linear least squares fit of

$$
y=0.8 x-150,
$$

Root Mean Squares Error 10.3, $R^{2} 0.615$, p-value 6.35e-20, and is strongly associated with the physical components of the dialysis procedure (pump, pump speed, dialysis line set), with variance from the line fit dominated by unmodeled physiological and mechanical effects. In order to further examine this hypothesis, it is possible to negate the time varying effects in the data by taking a mean value for each individual patient treatment, and recalculating the data scatter, and is shown in Fig. 3. A linear least squares fit of $y=$ $0.82 x-1.5 e+02$, Root Mean Squares Error 6.54, $R^{2}$ 0.852, p-value 2.93e38. There are a number of salient features in this plot. Firstly, the gradient 


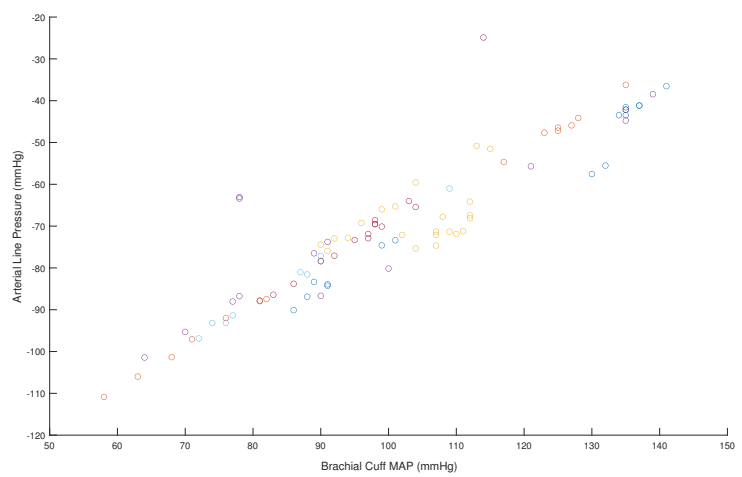

Figure 3: Brachial cuff MAP vs Arterial line pressures in 11 patient sessions, following compensation for time varying effects during treatment

of the fit has only changed by $2.5 \%$, indicating a relatively accurate model for the machine and lines system. Secondly, the data $R^{2}$ value has risen by $38.5 \%$ to 0.852 , indicating that it should be possible to design a tractable and useable time-invariant model. The feasibility study confirmed that it is potentially possible to estimate brachial $\mathrm{BP}$ continuously and non-invasively during the period of a dialysis session in a clinical setting with measurement of venous and arterial line pressure, and occasional measurement of brachial blood pressure by arm cuff for calibration.

\subsection{Structure of this paper}

This paper describes the development, application and performance analysis of an adaptive model parameter optimisation scheme based on iterative run-to-run learning control. The aim of this development was to compensate for the time-dependent changes of model parameters which describe the arterial line to BP relationship during 4-hour dialysis treatments. The paper is divided into the following sections:

- Section I: Introduction

- Section II: Run-to-run BP Estimator describes the application of runto run iterative learning methodology

- Section III: Method describes the processing methodology for the realtime patient data sets 
- Section IV: Results examines the effectiveness of the BP estimation scheme

- Section V: Conclusions assesses the overall modeling-predictor method and draws conclusions for further work

For clarity, where a patient's BP is mentioned, this should be taken to mean brachial blood pressure.

\subsection{Novelty and Contribution to Knowledge}

As noted in the introduction, a small number of technologies exist which can to a certain extent achieve BP monitoring during dialysis treatment. However, they share shortcomings with respect to sensitivity to patient movement, may cause patient discomfort, and are unreliable with patients with compromised blood flow in the extremities (a common problem for patients with ESK). Continuous BP monitoring over the entire 4-hour treatment time would, if combined with an accurate hypotension onset predictor create the means to implement closed loop control to achieve pro-active treatment regimes. Direct measurement of fluid pressure waveforms in the dialysis lines are suitable for continuous measurement during dialysis treatment due to their non-invasive nature, insensitivity to patient movement, and lack of reliance on sensors placed on the patient's extremities. Additionally a feasibility study conducted by the authors (20) has shown that it is possible to derive a mathematical relationship between brachial $\mathrm{BP}$ and the pressures measured in the dialysis lines. The current state-of-the-art in this area is relatively sparse. In (19), analysis of finger pulse waves, obtained from O2 sensors, estimates a beat-to-beat systolic pressure value. However, as in other methods described above, it is noted that the accuracy of estimation is significantly compromised by patient movement. The use of pressure signals from the arterial and venous blood lines of a dialysis machine have been shown in principle to enable continuous online monitoring of a patient's heart rate, even for patients with low cardiac signal amplitude $(21 ; 22)$. The method has been extended to monitor ventricular premature beats (23). Performance was studied for one dialysis flow rate $(400 \mathrm{ml} / \mathrm{min})$, and results lacked fidelity where patient heart rate and haemodialysis pump speed coincide. While promising, the method generated to a number of false positive and false negative predictions of IDH. Novelty and contribution to knowledge presented in this paper can be summarised as follows: 
- Continuous monitoring is achieved irrespective of patient movement and/or compromised blood flow

- A method which is completely non-invasive and does not compromise patient comfort

- A method which can reliable operate for the entire duration of treatment sessions

- This paper describes a novel inter-disciplinary application of Control Engineering via Run to Run Learning methodology.

- The method allows calibration during treatment via an occluding arm cuff

- Application of an adaptive methodology which tracks physiological changes during treatment

- The paper describes a novel, key enabling step forward as the continuous data stream which will be required in order to develop a BP real-time predictor.

Our approach demonstrates a more robust approach to derive continuous BP signals from the arterial and venous dialysis lines and presents the initial results of its in-vivo verification via on-going patient studies in the Renal Unit at Royal Derby hospital.

\section{Run-to-Run BP Estimator}

The system under consideration (Fig.4) is discrete-time and non-stationary, with continuous dialysis line pressures sampled at $1 \mathrm{kHz}$. This measurement constitutes the basis of calculations to estimate instantaneous, real-time BP. Patient arterial pressure is measured by occluding arm-cuff with an irregular sample rate of approximately 30 minutes, and this constitutes an accurate, repetitive measurement of $\mathrm{BP}$, albeit at extremely widely spaced timeinstances. The challenge addressed in this paper is to devise a method to, as accurately as possible, 'fill in the gaps' between BP cuff measurements. This is critically important, as patient BP can vary significantly at the time-scale of 30 minute measurement samples. 
For processes such as this, which are fundamentally repetitive or cyclical by nature and resemble a batch process delineated by cuff measurements, iterative learning is generally a tractable approach to modeling and control (26). This approach is generally applied to the problem of repetitive reference tracking control for example in the manufacturing of semiconductors (27). In the application described here, the methodology (Fig. 4) will be adapted to form a model-based BP estimator. Estimation of BP is based on measured arterial line pressure, however, an accurate measurement of BP to update the model parameters is only infrequently available (at best every 30 mins). Run-to-run control was developed during the 1990s $(28 ; 29)$ for this class of repetitive batch process. The model structure can take any form, however linear regressions and response surface structures are generally implemented (30), which assume that the process drift is slow enough to compensate with parameter updates when measurements become available. In this application, a model parameter update becomes available at the instant of BP cuff measurement.

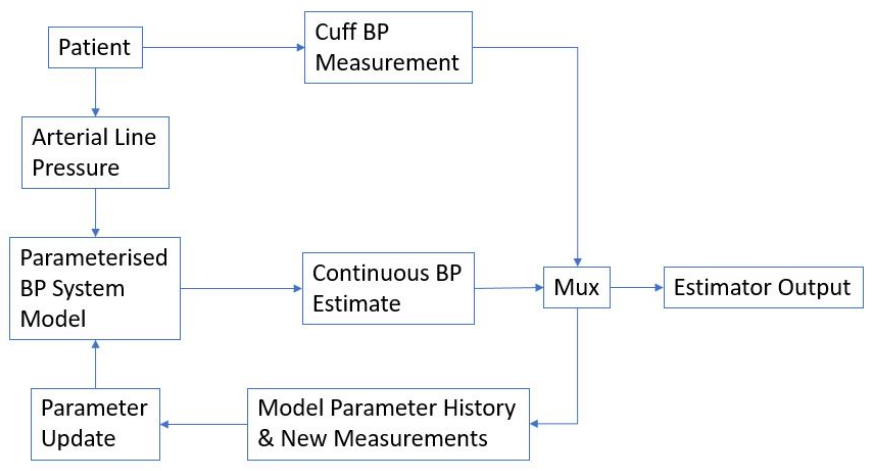

Figure 4: BP estimator system

A linear regression model of the form

$$
y(t)=a x(t)+b(t)+\epsilon(t)
$$

is generally implemented, where $t=1,2, \ldots$ denotes the time index; $y$ is the model output, estimated BP in $\mathrm{mmHg} ; x$ is the input, measured arterial line pressure in $\mathrm{mmHg} ; a$ is the slope coefficient; $b$ is the drift coefficient, and $\epsilon$ is the disturbance coefficient. The update objective for $y$ is $y^{*}$, and if $a$ and $b$ are accurately known, then the optimal model for the system is

$$
x(t)=a^{-1}\left(y^{*}-b(t)\right) .
$$


Model inaccuracies when compared to the system are inevitable, particularly given the non-stationary nature of the physiological process, unmodeled nonlinearities and stochastic events. Consequently, an iterative parameter adjustment is implemented to update the estimation of $a$ and $b$

$$
b(t)=\lambda[y(t-1)-a x(t-1)]+((1-\lambda) b(t-1))
$$

which is an exponentially weighted moving average filter (27), tuned by adjusting variable $\lambda$ in the interval $0<\lambda<1$. Combining (4) with (3) gives

$$
\begin{aligned}
& b(t)=\lambda\left[y(t-1)-a a^{-1}\left[y^{*}-b(t-1)\right]\right]+(1-\lambda) b(t-1) \\
& b(t)=b(t-1)+\lambda\left[y(t-1)-y^{*}\right]
\end{aligned}
$$

which, in its general form is

$$
x(t)=\alpha x(t-1)-r(t)
$$

where $0<\alpha<1$ and $r(t)$ are respectively the forgetting factor and update law for the model. Typically the system gain $a$ is modeled in advance via designed experiment (31). In the case under consideration here, the fitted linear relationship for the study population has been derived (eq.1) as $y=$ $0.8 x-1.5 e+02$ where $x$ is the measured variable Brachial cuff $B P$ and $y$ is the corresponding output variable Arterial Line Pressure. In the specific case under examination here, the system gain $a$ has been calculated from the study population linear fit, the estimator operates as follows:

- At startup, arterial BP is estimated from arterial line pressure via the study population linear fit. $(x(t-1)$ in Eqn.6)

- When a new BP measurement becomes available from a cuff measurement at time $t$, a new fit line is created through the updated cuff-line pressure point pair at gradient $a$

- A new estimator model is generated by the update law $r(t)$ in Eqn.6) which generates a new model line parallel to, and between the model line at $t-1$ and the new fit line at time $t$. The forgetting factor $\alpha$ defines the position of the new model line with respect to the line at $t-1$ and the new fit line at $t$ 
- In this case $\alpha$ is 0.5

- The method is repeated from item 2 whenever a new BP cuff measurement becomes available

An example of real-time operation of this algorithm is shown in Fig.(9) Given its successful history in the design and implementation of controllers for batch processes, the methodology has been applied to the design of a real-time BP estimator, which is described in the next section.

\section{Method}

In total 12 participants were recruited with 11 monitored dialysis sessions. 1 participant moved out of area before a session could be conducted. Of the 11 sessions, 4 monitoring data sets were incomplete due to a combination of circulatory and/or hypotensive episode during treatment rendering the Finapres data insufficient for our requirements. Consequently two of our patient group agreed to a further monitoring session each which were completed before lockdown was enforced due to COVID-19 in the UK. 9 complete 4-hour data sets were thus available for analysis.

$58.3 \%$ of participants were male, with a median age of 65 (IQR 48-78). All participants had continuous non-invasive monitoring of haemodynamics using pulse wave analysis (Finapres NOVA) during a single dialysis treatment of typically 4 hours duration, and data periodically verified by intermittent arm-cuff measurements. Two pressure sensors were attached to the dialysis lines: one between the arterial dialysis needle and dialysis line and the other directly onto the venous line bubble trap. Data was continuously recorded from all sensors at a sampling rate of $1 \mathrm{kHz}$.

The study protocol was approved by the West Midlands - Coventry and Warwickshire Research Ethics Committee and participants gave written informed consent.

A typical real-time data set for a patient study is shown in Fig.5. Measurements taken during the observational study were acquired and stored for each patient as data sets containing the following datastreams covering the entire treatment period, typically 4 hours;

- Time: continuous from National Instruments board

- Arterial line pressure: continuous from pressure sensor 
- Venous line pressure: continuous from pressure sensor

- Occluding cuff BP: intermittent from Finapres Nova

- Reconstructed systolic, diastolic and mean arterial pressure: continuous from Finapres Nova finger cuff

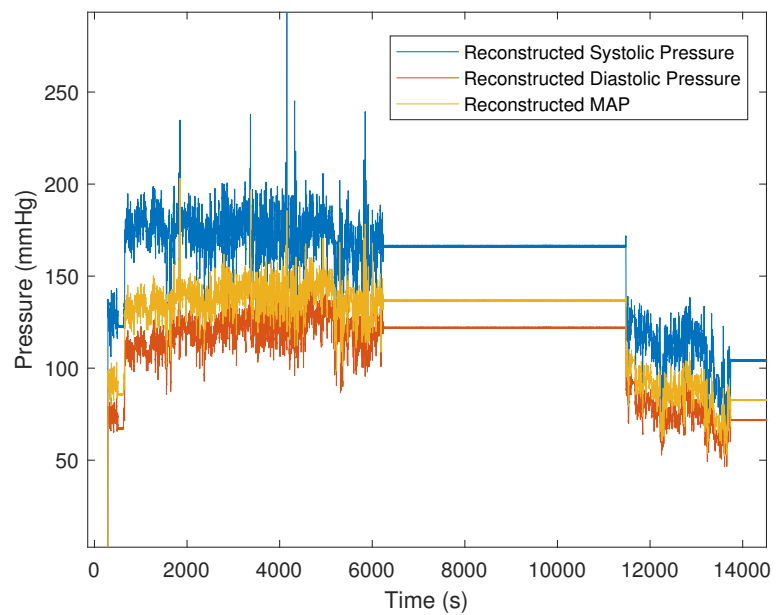

Figure 5: Typical patient real-time data - reconstructed systolic, diastolic and mean arterial pressure (IT54)

We will now consider the data from a single typical treatment session for patient IT54. Fig. (5) shows the reconstructed systolic, diastolic and mean arterial pressures calculated by the Finapres Nova unit from a vascular unloading finger cuff. It is found in practice that this method causes patient discomfort which generally precludes continuous use, hence in this case approximately 2 hours of data at the beginning of treatment and 30 mins of data at the end of treatment are recorded. This was the case for all of the participants in the study, with 30 minute sessions being the average period for finger cuff measurements before the device causes discomfort. Additionally, as the cuff is fitted to a finger on the non-dialysing arm, it can also interfere with run-of-the-mill activities such as refreshment breaks and reading.

Fig.(6) shows the directly measured venous and arterial line pressures, along with estimated blood pump flow rate which is derived from the venous line pressure data. 


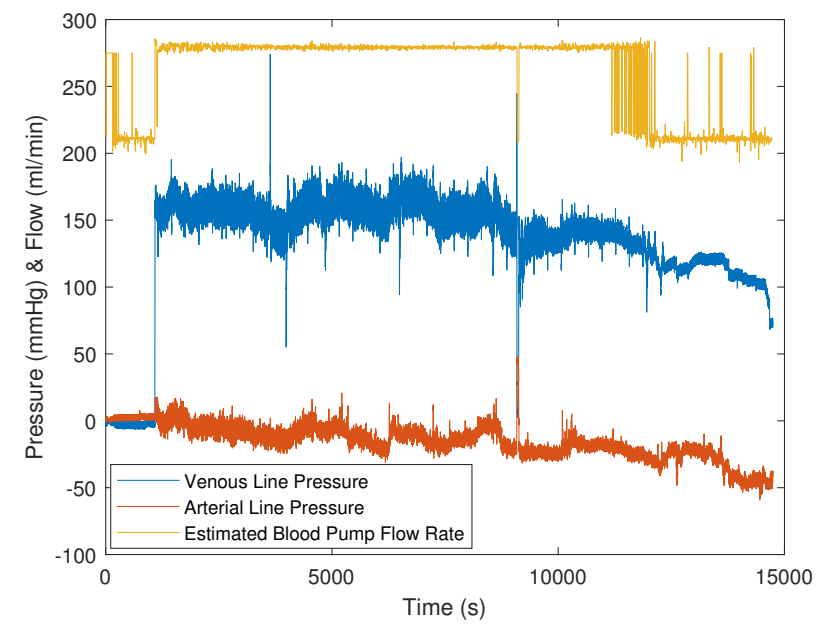

Figure 6: Typical patient real-time data - venous and arterial line pressure, estimated blood pump flow rate (IT54)

Fig.(6) reveals a venous line pressure waveform which is time varying with respect to both mean and amplitude. This measurement is taken at a port on the venous bubble trap which is situated close to the dialyser blood pump. As is to be expected, the waveform dynamics are dominated by the action of the two lobes of this peristaltic pump Fig.(7). This dominating waveform shape should be closely related to the angular speed of the pump and hence should support the derivation of flow rate through the dialysis lines. The time varying nature of the waveform renders this derivation potentially problematic, leading to the application of Fourier modelling to calculate the fundamental frequency of the pump. This method supports the derivation of instantaneous, and hence, time varying pump speed (32). The problem can be posed as a moving window length $L$ over the real-time venous line pressure data stream which is periodic over the interval $0 \geq x \leq L$, then $f(x)$ can be derived as

$$
f(x)=a_{0}+\sum_{n=1}^{\infty}\left[a_{n} \cos \left(\frac{2 \pi n x}{L}\right)+b_{n} \sin \left(\frac{2 \pi n x}{L}\right)\right]
$$

which is the Fourier trigonometric series for the function $f(x)$. In the case under examination here, the fundamental frequency of the blood pump is sought, so calculations are constrained to $n=1$. 


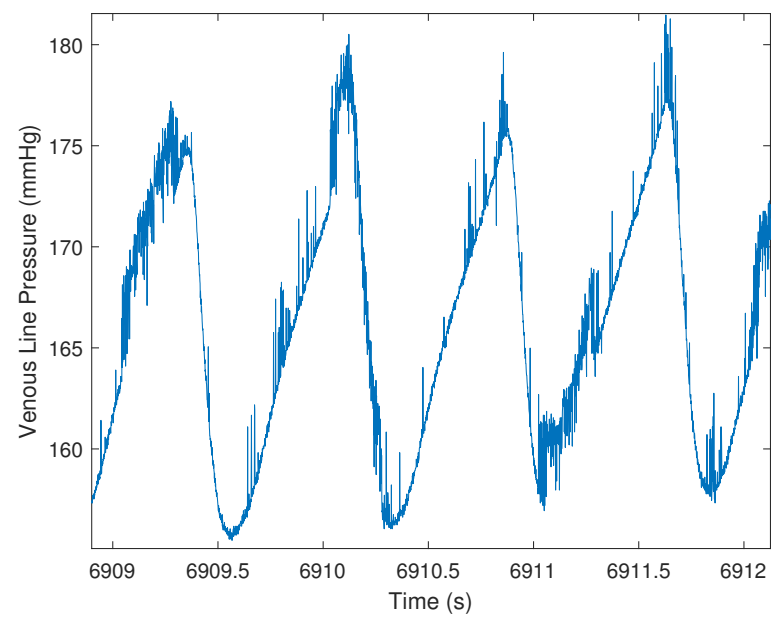

Figure 7: Typical patient real-time data - venous line pressure detail (IT54)

Examination of Figs.(5-7) reveals waveforms which comprise cyclical elements which are varying in both amplitude and mean in addition to measurement noise. In order to smooth the signals to facilitate real time calculations, moving average filters with a window length of 5000 samples or $5 s$ was implemented on all data lines with the exception of BP which has a single value at each measurement event. The window length was chosen as being over twice the length of the period of a slow heart rate (HR) of $30 \mathrm{bpm}$ in order to avoid aliasing. The pump frequency is calculated and updated in real-time over this sliding window. Additionally, arm cuff measurements are characterised as steady-state phenonomena as they are discrete measurements without dynamics. Derived pump frequency is converted to flow in units of $\mathrm{mls}^{-1}$ by

$$
f l=\left(\left(\frac{f r\left(r a d s^{-1}\right)}{2 \pi}\right) \times 60\right) \times\left(\pi r^{2} L n\right)
$$

where $f l$ is flow in $m l s^{-1}, f r$ is pump frequency in $r a d s^{-1}, r$ is the radius of the dialysis line within the pump in $m m$ and $L n$ is the effective length of line within the pump in $\mathrm{mm}$. In the example shown in Fig.(6) the estimate of $290 \mathrm{ml} / \mathrm{min}$ agrees with the flow rate recorded in the patient's record.

Filtered mean arterial line pressure and reconstruced arterial waveforms are shown in Fig.(8) with corresponding arm cuff MAP measurements during 


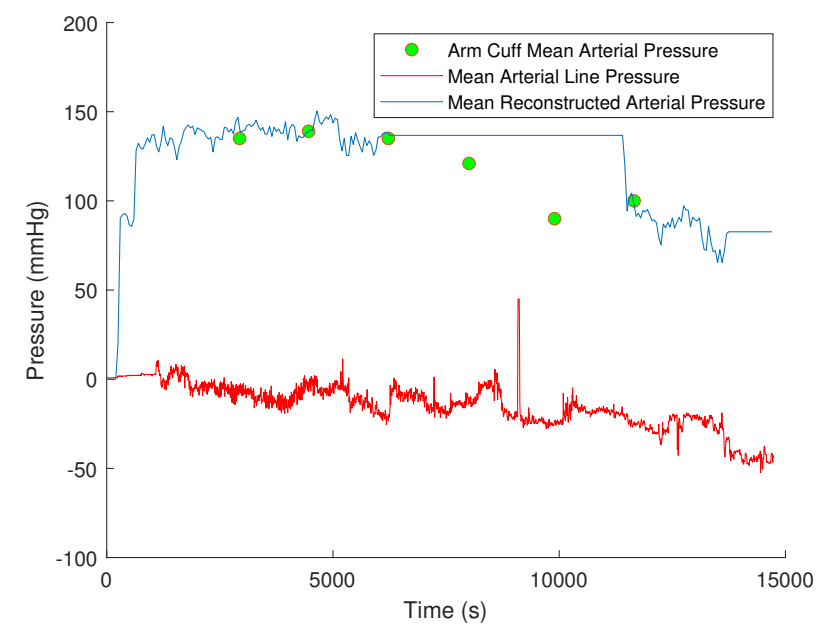

Figure 8: Typical patient real-time data - occluding arm cuff pressure, mean arterial line pressure, mean reconstructed arterial pressure (IT54)

the treatment period.

Real-time code has been developed in Matlab/Simulink (33) to perform calculations on patient measurement data streams acquired during dialysis treatment. The algorithm is structured as follows;

1. pre-calculate recursive least fit for the patient populations previously measured and recorded BP - arterial line pressure pairs Fig.(2). In this case, with $x$ as the measured variable arterial line pressure and $y$ as the predictor variable $B P$, we derive the relationship of the form $y(t)=a x(t)+b(t)+\epsilon(t))$ Eq. 2 as $y=0.619 x+138.8+\epsilon$

2. the $a$ coefficient in Eq.(2) is thus fixed as 0.619 for this patient and will be updated for future treatments as more data is acquired

3. initial estimates of $\mathrm{BP}$ are conducted with the population derived fit until the first new $\mathrm{BP} /$ line pressure measurement is received

4. the relationship $y(t)=a x(t)+b(t)+\epsilon(t))$ is updated according to Eq. 4

5. estimates of BP are conducted with the updated estimator

6. new measurements received

7. repeat from item 4 
Table 1: Baseline participant characteristics

\begin{tabular}{|c|c|}
\hline VARIABLE & $(\mathrm{n}=7)$ \\
\hline Age (years) & $53\left({ }^{*}\right.$ IQR 49 to 70$)$ \\
\hline Male [n (\%)] & $3(43)$ \\
\hline White ethnicity [n (\%)] & $5(71)$ \\
\hline Current smoking [n (\%)] & $1(14)$ \\
\hline Diabetes [n (\%)] & $2(29)$ \\
\hline Ischaemic heart disease [n (\%)] & $2(29)$ \\
\hline Dialysis vintage (months) & 6 to 23$)$ \\
\hline Dialysis adequacy (Kt/V) & $1.24(0.97$ to 1.46$)$ \\
\hline Fistula blood flow assessment $(Q A)$ & $670(635$ to 1072$)$ \\
\hline Vascular access type & \\
\hline Brachiocephalic arteriovenous fistula [n (\%)] & $4(57)$ \\
\hline Radiocephalic arteriovenous fistula [n (\%)] & $3(43)$ \\
\hline Needle gauge & $3(43)$ \\
\hline 2x 14g [n (\%)] & $3(43)$ \\
\hline 2x 15g [n (\%)] & $1(14)$ \\
\hline Arterial 15g; Venous 16g [n (\%)] & $78.6(64.1$ to 98.2$)$ \\
\hline Post-dialysis weight $(\mathrm{kg})$ & $26.3(24.4$ to 34.4$)$ \\
\hline Body mass index $\left(\mathrm{kg} / \mathrm{m}^{2}\right)$ & \\
\hline *IQR, interquartile range & \\
\hline \multicolumn{2}{|c}{}
\end{tabular}

\section{Results}

9 complete treatment data sets were processed and subsequently analysed, characteristics of the patients in the study are given in Table 1

Fig.9 shows the typical updating of the model over an approximately 4hour treatment period as new $\mathrm{BP} /$ line pressure measurements are acquired.

The associated BP estimator for this treatment is shown in Fig.10. Blood pump flow for this treatment is consistent at $280 \mathrm{ml} / \mathrm{min}$.

The initial estimate at 3500s is based upon the initial population fit model and exhibits a relatively wide divergence from the incoming accurate BP measurement. Subsequent pairs show the incoming BP measurement, with the estimate from the predictor model prior to update, and exhibit improved accuracy when compared to the first estimate in all patients.

Performance of the estimator scheme for patients in the study is shown in 


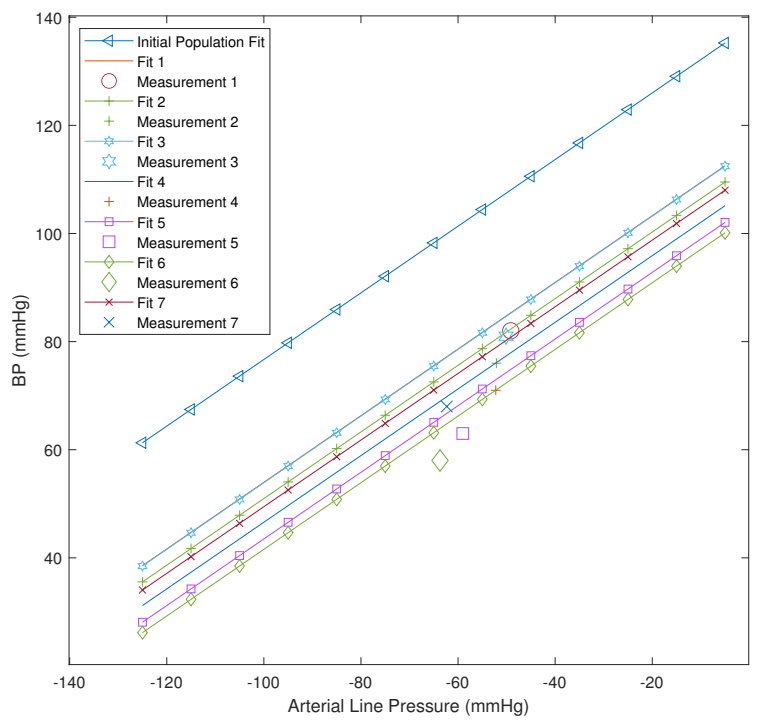

Figure 9: IT62 Initial population fit predictor, incoming measurements and predictor updates over 4 hour treatment

Table 2. Maximum errors are exclusively associated with the initial estimates derived from the population model. Errors then tend to decrease significantly after the first BP update. The average RMS error for all the sessions analysed here (including initial modelling error) is $11.5 \mathrm{~mm} \mathrm{Hg}$. Eliminating the initial modeling error reduces the average RMS error to $7.9 \mathrm{~mm} \mathrm{Hg}$

Finally for completeness, we can examine the performance of the estimator against dynamic, continuous BP measurement (Fig. 11). There is reasonable performance in tracking the cuff BP measurements, however, with the continuous BP measurement provided by the Fiapres Nova finger cuff there are obvious shortcomings. This is to be expected when comparing the performance of a steady-state linear model against the output of a dynamic non-linear system.

\section{Conclusion}

A non-invasive, recursive BP estimator has been designed and analysed on real-time data sets from an observational patient study. The parameterised run-to-run adaptive model described is initially fitted to data from 


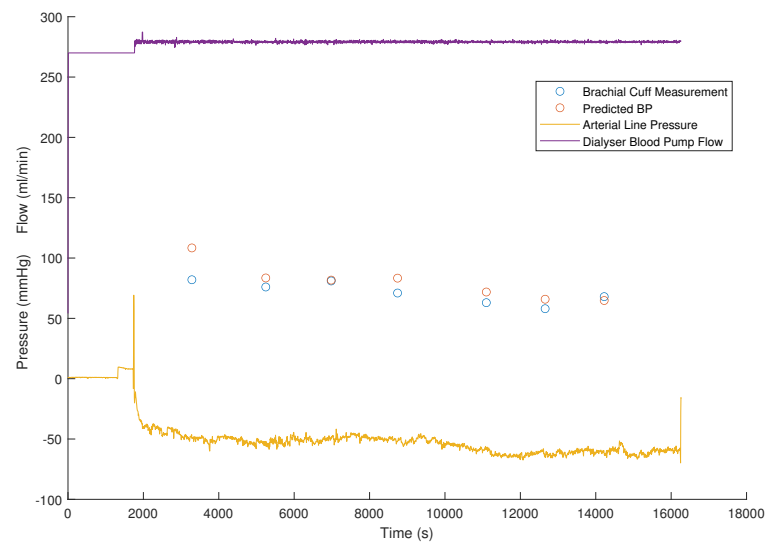

Figure 10: IT62 measured and predicted BP, arterial line pressure and blood pump flow over 4 hour treatment

the study population, and subsequently updated with the individual's line and $\mathrm{BP}$ data when it is available. The problem of defining initial conditions for the estimator model and reliance on sparse update data is extremely challenging. There are a number of significant outcomes from this phase of the research programme. Firstly, in this study, and a previous 50 patient study which examined heart rate and blood pressure variability in dialysis treatment (34), it is apparent that existing continuous monitoring technology currently faces challenges in applications with long treatment times. The method described in this paper successfully conducts estimation of BP based only on the pressure measurements from sensors placed in the dialysis lines, and intermittent BP measurements. As such it fulfills the requirements of a usable non-invasive, continuous estimator.

\subsection{Discussion}

The hypothesis of the work described in this paper is that a method could be developed which demonstrates continuous non-invasive blood pressure monitoring, without compromising patient experience and without requiring bespoke interfacing to the dialysis machine, or any additional sensing to be worn by the patient beyond current arm cuff monitoring requirements. A generalised mathematical model relating arterial line pressure, blood pump flow rate and brachial pressure has previously been developed by the authors, which predicts a quasi-linear relationship between arm cuff measured pres- 
Table 2: Estimator performance over treatment sessions when compared to incoming BP measurements ( $\mathrm{mmHg})$

\begin{tabular}{|c|c|c|c|}
\hline Patient & RMS Error & Max Error & Min Error \\
\hline IT62 & 12.27 & 26.38 & 0.71 \\
\hline IT58 & 7.47 & 14.89 & 2.08 \\
\hline IT57 & 11.19 & 21.17 & 0.38 \\
\hline IT54 & 28.48 & 51.45 & 1.7 \\
\hline IT62 & 11.51 & 28.68 & 0.38 \\
\hline IT60 & 7.47 & 18.9 & 1.22 \\
\hline IT57 & 12.94 & 25.68 & 1.06 \\
\hline IT56 & 6.76 & 13.08 & 0.77 \\
\hline IT55 & 5.56 & 14.33 & 2.01 \\
\hline
\end{tabular}

sure, and corresponding pressure measured in the arterial line near to the fistula. The observational study presented in this paper derives continuous measurement of brachial pressure from continuous measurements of arterial and venous line pressures for the first time, via an empirically based mathematical model which is updated by a novel application of an Iterative Run to Run Learning algorithm derived from Control Engineering.

The study presented here confirms the fundamental principles of this approach, and the results have confirmed that the methodology and technology has a practical future in renal units. There are a number of associated challenges which will be addressed in future studies. In particular, the iterative learning algorithm presented here will be combined with improved mathematical models to increase estimation accuracy, and predictive models for hypotension. Patient studies are about to start at Royal Derby Hospital to demonstrate, analyse and improve the continuous estimation of brachial BP in real-time for the complete duration of dialysis treatments.

In its current form, the estimator can track trends in patient BP albeit with an average RMS error of $7.9 \mathrm{mmHg}$ between prediction and updated BP measurement. Consequently, it represents a significant step forward in this area as the ability to repeatably track changes in BP rather than measure instantaneous BP with high levels of accuracy is currently a critical necessity during treatment. Examination of Fig.11 reveals the mismatch between the performance of the linear steady-state model and the non-linear dialyser-physiological patient dynamics. Future work will develop a nonlinear parameterised model estimator, which will still utilise measured BP 


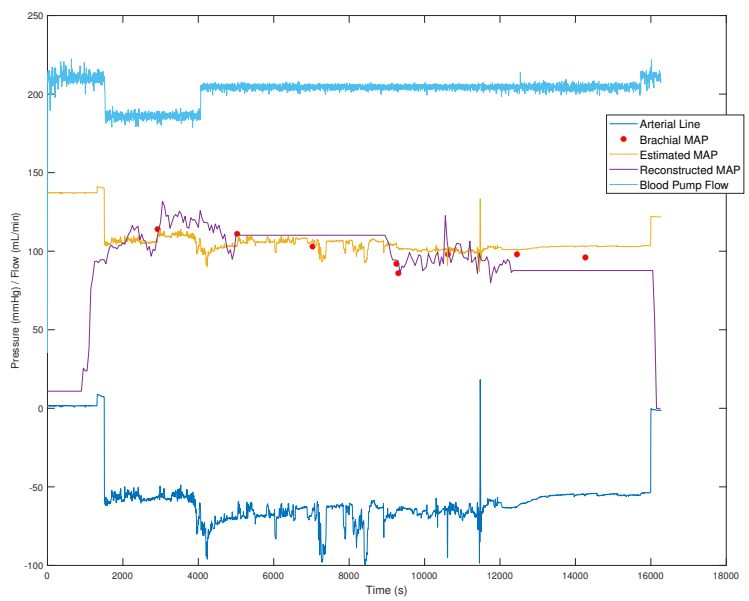

Figure 11: IT57 measured and predicted static and dynamic BP, arterial line pressure and blood pump flow over 4 hour treatment

updates, but will have more reliance on the complex interaction between the venous and arterial lines real-time pressure measurements. In this way, the model will have less reliance on a population model for initial conditions, and in particular will be able to estimate both steady-state and dynamic BP response, which should enable more accurate real-time estimates rather than trend estimation from the current estimation scheme.

\section{Acknowledgments}

The authors would like to thank Mel Morris and iTrend Medical Research Ltd. for funding the iTrend research programme, and also the support of the Royal Derby Hospital Renal Unit.

US Patent Office \& Overseas License 62/855069 MEASURING PRESSURE WAVES IN DIALYSIS LINES TO DERIVE CONTINUOUS ARTERIAL BLOOD PRESSURE Prof Paul Stewart, Prof Jill Stewart, et al. iTrend Medical Research Ltd. 


\section{References}

[1] A. Meidert and B. Saugel, Techniques for Non-Invasive Monitoring of Arterial Blood Pressure, Front Med (Lausanne). (2017); 4: 231. Published online 2018 Jan 8. doi: 10.3389/fmed.2017.00231.

[2] J. Bijker, S. Persoon, L. Peelen, K. Moons, C. Kalkman, L. Kappelle, et al. Intraoperative hypotension and perioperative ischemic stroke after general surgery: a nested case-control study, Anesthesiology (2012) 116:658-64.10.1097/ALN.0b013e3182472320

[3] J. van Waes, W. van Klei, D. Wijeysundera, L. van Wolfswinkel, T. Lindsay, W. Beattie, Association between intraoperative hypotension and myocardial injury after vascular surgery, Anesthesiology (2016) 124:35-44.10.1097/ALN.0000000000000922

[4] M. Walsh, P. Devereaux, A. Garg, A. Kurz, A. Turan, R. Rodseth, et al., Relationship between intraoperative mean arterial pressure and clinical outcomes after noncardiac surgery: toward an empirical definition of hypotension, Anesthesiology (2013) 119:507-15.10.1097/ALN.0b013e3182a10e26

[5] Flythe et. al. Association of mortality risk with various definitions of intradialytic hypotension, J Am Soc Nephrol. (2015) Mar; 26(3):72434. doi: 10.1681/ASN.2014020222. Epub 2014 Sep 30.

[6] G. Mahe, E. Comets, A. Nouni, F. Paillard, C. Dourmap, A. Le Faucheur and V. Jaquinandi, A minimal resting time of twenty five minutes is needed before measuring stabilized blood pressure in subjects addressed for vascular investigations, Scientific Reports volume 7, Article number: 12893 (2017)

[7] G. Pressman and P. Newgard, A transducer for the continuous external measurement of arterial blood pressure, IEEE Trans Biomed Eng (1963) 10:73-81.

[8] A. Meidert, W. Huber, A. Hapfelmeier, M. Schöfthaler, J. Müller, N. Langwieser, et al., Evaluation of the radial artery applanation tonometry technology for continuous noninvasive blood pressure monitoring compared with central aortic blood pressure measurements in patients 
with multiple organ dysfunction syndrome, J Crit Care (2013) 28:908-12.10.1016/j.jcrc.2013.06.012

[9] B. Saugel, R. Dueck and J. Wagner. Measurement of blood pressure, Best Pract Res Clin Anaesthesiol (2014) 28:309-22.10.1016/j.bpa.2014.08.001

[10] R. Dueck, O. Goedje and P. Clopton. Noninvasive continuous beat-tobeat radial artery pressure via TL-200 applanation tonometry, J Clin Monit Comput (2012) 26:75-83.10.1007/s10877-012-9336-2

[11] J. Penaz, A. Voigt and W. Teichmann. Contribution to the continuous indirect blood pressure measurement, Z Z Gesamte Inn Med (1976) 31:1030-3

[12] Wang R, Jia W, Mao ZH, Sclabassi RJ, Sun M., Cuff-Free Blood Pressure Estimation Using Pulse Transit Time and Heart Rate, Int Conf Signal Process Proc. (2014) ;2014:115-118. doi:10.1109/ICOSP.2014.7014980

[13] Meidert Agnes S., Saugel Bern, Techniques for Non-Invasive Monitoring of Arterial Blood Pressure Frontiers in Medicine, 5, 231, (2018).

[14] C. Rhee, Y. Obi, A. Mathew and K. Kalantar-Zadeh, Precision Medicine in the Transition to Dialysis and Personalized Renal Replacement Therapy, Semin Nephrol. (2018) Jul;38(4):325-335. doi: 10.1016/j.semnephrol.2018.05.003.

[15] G. Piccoli, L. Nielsen, L. Gendrot, A. Fois, E. Cataldo and G. Cabiddu, Prescribing Hemodialysis or Hemodiafiltration: When One Size Does Not Fit All the Proposal of a Personalized Approach Based on Comorbidity and Nutritional Status, settings J. Clin. Med. (2018), 7(10), 331; https://doi.org/10.3390/jcm7100331

[16] R. Agarwal, T. Metiku, G. G. Tegegne, R. P. Light, Z. Bunaye, D. Bekele and K. Kelley, Diagnosing Hypertension by Intradialytic Blood Pressure Recordings, Clin J Am Soc Nephrol. (2008) Sep; 3(5): 1364-1372. doi: 10.2215/CJN.01510308

[17] M. Assimon, and J. Flythe, Intradialytic blood pressure abnormalities: the highs, the lows, and all that lies between, Am J Nephrol. (2015); 42(5): 337-350. Published online 2015 Nov 20. doi: 10.1159/000441982 
[18] Stewart, Jill, Paul Stewart, Thomas Walker, Tarek Eldehini, Daniela V. Horner, Bethany Lucas, Kelly White, et al. (2020). A Feasibility Study of Non-invasive Continuous Estimation of Brachial Pressure Derived from Arterial and Venous Lines During Dialysis engrXiv. May 7. doi:10.31224/osf.io/xpv65

[19] Mambelli E, Mancini E, Santoro A. A continuous and non-invasive arterial pressure monitoring system in dialysis patients. Nephron Clin Pract. 2007;107(4):c170-6. doi: 10.1159/000110677. Epub 2007 Nov 1. PMID: 17975324 .

[20] J. Stewart, P Stewart et al., "A Feasibility Study of Non-invasive Continuous Estimation of Brachial Pressure Derived from Arterial and Venous Lines During Dialysis," (2020) IEEE Journal of Translational Engineering in Health and Medicine, doi: 10.1109/JTEHM.2020.3035988.

[21] M. Holmer, F. Sandberg, K. Solem, E. Grigonyte, B. Olde and L. Sornmo, Extracting a Cardiac Signal From the Extracorporeal Pressure Sensors of a Hemodialysis Machine, IEEE Transactions on Biomedical Engineering, vol. 62, no. 5, pp. 1305-1315, May (2015).

[22] M. Holmer, F. Sandberg, K. Solem, B. Olde and L. Sornmo, Heart Rate Estimation from Dual Pressure Sensors of a Dialysis Machine, Computing in Cardiology (2015); 42:29-32, ISSN 2325-8861 .

[23] M. Holmer, J. Martínez, E. Gil, F. Sandberg, B. Olde and L. Sörnmoa, Detection of ventricular premature beats based on the pressure signals of a hemodialysis machine, Medical Engineering \& Physics, Volume 51, January (2018), Pages 49-55, https://doi.org/10.1016/j.medengphy.2017.11.004.

[24] P. Stewart, J. Stewart, T. Walker, L. Gullapudi, N. Selby and M. Taal, Measuring pressure waves in dialysis lines to derive continuous arterial blood pressure: pilot work in an in vitro and in silico model Nephrology Dialysis Transplantation, Volume 34, Issue Supplement 1, June (2019), gfz103.SP541, https://doi.org/10.1093/ndt/gfz103.SP541 Published: 13 June 2019

[25] P. Stewart, J. Stewart, T. Walker, L. Gullapudi, M. Taal and N. Selby, Development of an in vitro simulation model to investigate haemody- 
namic responses during haemodialysis, Nephrology Dialysis Transplantation, Volume 34, Issue Supplement 1, June (2019), gfz106.FP630, https://doi.org/10.1093/ndt/gfz106.FP630 Published: 13 June 2019

[26] Youqing Wang, Furong Gao and Francis J.Doyle III, Survey on iterative learning control, repetitive control, and run-to-run control, Journal of Process Control, Volume 19, Issue 10, December (2009), Pages 15891600, https://doi.org/10.1016/j.jprocont.2009.09.006

[27] Moyne J. (2014) Run-to-Run Control in Semiconductor Manufacturing. In: Baillieul J., Samad T. (eds) Encyclopedia of Systems and Control. Springer, London

[28] E. Sachs, R.-S. Guo, S. Ha and A. Hu, On-line process optimization and control using the sequential design of experiments, Digest of Technical Papers.1990 Symposium on VLSI Technology, Honolulu, Hawaii, USA, (1990), pp. 99-100, doi: 10.1109/VLSIT.1990.111027.

[29] E. Sachs, R.-S. Guo, S. Ha and A. Hu, Process control system for VLSI fabrication," IEEE Transactions on Semiconductor Manufacturing, vol. 4, no. 2, pp. 134-144, May (1991), doi: 10.1109/66.79725.

[30] William Jarrett Campbell, Stacy Firth, Anthony Toprac, Thomas F. Edgar, A comparison of Run-to-Run Control algorithms, (2002) Proceedings of the American Control Conference 3:2150 - 2155 vol.3 DOI: 10.1109/ACC.2002.1023955

[31] G.P.Box, W.G. Hunter and J.S. Hunter, Statistics for Experimenters: An Introduction to Design, Data Analysis, and Model Building, John Wiley and Sons Inc., New York, 1st ed., (1978).

[32] I. López-García, M. Sánchez-Merlos, P. Viñas, M. Hernández-Córdoba, Peristaltic pumps-Fourier transforms: a coupling of interest in continuous flow flame atomic absorption spectrometry, Spectrochimica Acta Part B: Atomic Spectroscopy, Volume 51, Issue 14, (1996), Pages 17611768, ISSN 0584-8547, https://doi.org/10.1016/S0584-8547(96)01557-1. (http://www.sciencedirect.com/science/article/pii/S0584854796015571)

[33] https://uk.mathworks.com/products/matlab.html 
[34] J. Stewart, P. Stewart, T. Walker, L. Gullapudi, T. Eldehni, N. Selby et al. (2020), An Application of The Lomb-Scargle Periodogram To Investigate Heart Rate Variability During Haemodialysis TechRxiv. Preprint. https://doi.org/10.36227/techrxiv.12442181.v1 